\title{
Wetting properties of structured interfaces composed of surface-attached spherical nanoparticles
}

\author{
Bishal Bhattarai and Nikolai V. Priezjev \\ Department of Mechanical and Materials Engineering, \\ Wright State University, Dayton, Ohio 45435
}

(Dated: September 10, 2018)

\begin{abstract}
The influence of the external pressure and surface energy on the wetting transition at nanotextured interfaces is studied using molecular dynamics and continuum simulations. The surface roughness of the composite interface is introduced via an array of spherical nanoparticles with controlled wettability. We find that in the absence of an external pressure, the liquid interface is flat and its location relative to the solid substrate is determined by the particle size and the local contact angle. With increasing pressure on the liquid film, the interface becomes more curved and the three-phase contact line is displaced along the spherical surface but remains stable due to re-entrant geometry. It is demonstrated that the results of molecular dynamics simulations for the critical pressure of the Cassie-Baxter wetting state agree well with the estimate of the critical pressure obtained by numerical minimization of the interfacial energy.
\end{abstract}

PACS numbers: 68.08.-p, 66.20.-d, 83.10.Rs 


\section{INTRODUCTION}

Over the last two decades, there has been a remarkable progress in designing and fabricating the so-called superhydrophobic surfaces, which are characterized by small-scale surface roughness that keeps the liquid interface suspended at the tips of the asperities, thus, reducing the liquid-solid contact area [1, 2]. Such engineered surfaces typically exhibit large liquid contact angles, small contact angle hysteresis, low adhesion, and reduced hydrodynamic friction, which play important roles in many technological processes, including self-cleaning surfaces, such as glasses and fabrics [3], nonfouling surfaces [4], and drag reduction [5]. Superhydrophobic textures can also enable a more accurate control and manipulation of liquid flows in microfluidic and nanofluidic systems [6] 8]. Notably, it was shown that laminar flows over anisotropic textured surfaces can be generally described via the mobility tensor that relates the normal traction at the interface and the effective slip velocity [9]. From a modeling perspective, a detailed comparison between continuum predictions and atomistic simulations demonstrated that there is excellent agreement between the velocity profiles and the effective slip boundary conditions if the length scales of surface patterns are large compared to the liquid molecular size [10, 11].

It is well known that, depending on the surface energy and topography, a liquid droplet in contact with a roughened substrate can form either the Wenzel state [12], where the liquid fully penetrates into the surface asperities, or the Cassie-Baxter state [13], where the liquid interface remains suspended at the tips of surface protrusions and small pockets of air become trapped between the surface and the liquid. In the latter case, the apparent contact angle is given by the Cassie-Baxter equation:

$$
\cos \theta_{c}=(1-f) \cos \theta-f
$$

where $f$ is the areal fraction of the liquid-air interface and $\theta$ is the intrinsic contact angle of a liquid droplet at a smooth surface of the same material. In particular, it follows from Eq. (1) that in the limit when the areal fraction $f$ is close to 1, the apparent contact angle becomes nearly $180^{\circ}$. In practice, however, the distance between surface protrusions should be sufficiently small, which allows the liquid-air interface to remain locally suspended above the surface; otherwise, the liquid would fully wet the substrate. Thus, the main factors that limit the applicability of superhydrophobic surfaces include the breakthrough pressure 
required to fully wet the substrate, fluid temperature, fragility of the surface texture, and fouling resistance [1].

More generally, the types of surfaces that exhibit highly repellent properties for liquids with both high and low surface tension (for example, water and oil) are called omniphobic surfaces [14, 15]. Such an unusual property can be achieved by introducing the re-entrant surface curvature, when the three-phase contact line is stabilized at the concave regions and the liquid interface remains suspended between surface asperities. The stability of the CassieBaxter state could be further improved in the case of hierarchical surface topography, where nanoscale texture is imposed on microscale roughness, and it might involve a combination of many concave and convex segments [16]. Recently, the equilibrium and stability of CassieBaxter wetting states on microstructured surfaces were analyzed by minimizing the surface free energy and the potential energy of the external pressure [17, 18]. An estimate of the critical value of the external pressure required to overcome the Laplace pressure due the curved interface was obtained for a number of typical 3D microstructures [17, 18]. In general, however, the shape of the liquid interface and the critical pressure for the transition from the Cassie-Baxter to Wenzel states on structured surfaces have to be determined numerically.

In recent years, the wetting behavior of liquid droplets on nanotextured surfaces was extensively studied using molecular dynamics (MD) simulations [19 29]. For instance, it was shown that the free-energy barrier associated with the transition from the Wenzel-to-Cassie state is much higher than from the Cassie-to-Wenzel state, provided that the height of pillars at the hydrophobic surface is sufficiently large [19]. It was also found that the apparent contact angle depends sensitively on the pillar cross-sectional shape and height, spacing between pillars, crystal plane orientation at the tops of the pillars, and the interaction energy between pillar atoms and droplet molecules [19, 22, 24, 26, 28]. Interestingly, during lateral motion of a liquid droplet over deformable pillar-arrayed substrate, the flexible hydrophilic pillars can accelerate the liquid upon approach and pin the receding contact line [23]. Furthermore, it was recently demonstrated that the wetting transition of a water droplet at a pillar-arrayed surface also depends on the charge density at the base substrate [26]. However, despite considerable computational efforts, the atomic details of the wetting transition at nanostructured surfaces with re-entrant surface curvature remain not fully understood.

In this paper, molecular dynamics and continuum simulations are preformed to investigate 
the effects of external pressure and surface energy on the wetting transition at nanotextured surfaces with re-entrant curvature. We consider a polymeric liquid film partially wetting the surface of a spherical particle, which is fixed rigidly at a solid substrate. It will be shown that the position of the liquid/vapor interface is determined by the particle radius and the local contact angle at the particle surface. With increasing external pressure on the liquid film, the liquid/vapor interface is displaced towards the substrate and becomes more curved until it touches the substrate at the critical pressure. We find an excellent agreement between the results of molecular dynamics simulations and numerical minimization of the surface energy for the critical pressure as a function of the local contact angle.

The rest of the paper is organized as follows. The description of molecular dynamics and continuum simulations is given in the next section. The relationship between the local contact angle and the surface energy for a liquid droplet on a flat substrate, and the pressure dependence of the shape and location of the suspended liquid film as well as the comparative analysis of the critical pressure as a function of the contact angle are presented in Sec. III. The results are summarized in the last section.

\section{SIMULATION DETAILS}

We study wetting properties of structured interfaces that consist of an array of spherical particles on a solid substrate and a suspended liquid film, as shown schematically in Fig.1. The large-scale molecular dynamics simulations were carried out using the parallel code LAMMPS developed at Sandia National Laboratories [30]. In our model, any two atoms interact via the truncated Lennard-Jones (LJ) potential

$$
V_{L J}(r)=4 \varepsilon\left[\left(\frac{\sigma}{r}\right)^{12}-\left(\frac{\sigma}{r}\right)^{6}\right]
$$

where the parameters $\varepsilon$ and $\sigma$ represent the energy and length scales of the liquid phase. The interaction between atoms of a liquid and a solid is also described by the LJ potential but with the parameters $\varepsilon_{\mathrm{wf}}$ and $\sigma_{\mathrm{wf}}$ that are measured in units of $\varepsilon$ and $\sigma$, respectively. The solid atoms are fixed on either lattice sites or on a surface of a sphere and they do not interact with each other. Furthermore, the size of liquid and solid atoms is chosen to be the same, i.e., $\sigma_{\mathrm{wf}}=\sigma$, throughout the study. For computational efficiency, the cutoff radius is set $r_{c}=2.5 \sigma$ for all types of interactions. 
We consider a polymeric fluid, where atoms are connected to form linear chains with $N_{p}=10$ atoms each, which makes the surface tension of the liquid/vapor interface greater than for monatomic liquids [38. In addition to the LJ potential, the interaction between nearest-neighbor atoms in a polymer chain is described via the FENE (finitely extensible nonlinear elastic) potential

$$
V_{F E N E}(r)=-\frac{k}{2} r_{o}^{2} \ln \left[1-r^{2} / r_{o}^{2}\right],
$$

with the parameters $k=30 \varepsilon \sigma^{-2}$ and $r_{o}=1.5 \sigma$ [32]. The combination of the LJ and FENE potentials with this parametrization yields an effective harmonic potential that allows vibration of nearest-neighbors but prevents polymer chains from unphysical crossing each other [32. We also comment that relatively short polymer chains considered in the present study form a polymer melt well below the entanglement limit of about 70 beads per chain [32]. The MD simulations were carried out at a constant temperature of $1.0 \varepsilon / k_{B}$, which was maintained via the Nosé-Hoover thermostat applied to the fluid phase [30]. Here, $k_{B}$ denotes the Boltzmann constant. At this temperature, the dependence of the surface tension at the liquid/vapor interface as a function of the chain length was reported in Ref. [38]. The equations of motion were solved numerically using the velocity Verlet integration algorithm [30, 31] with the time step $\triangle t_{M D}=0.005 \tau$, where $\tau=\sigma \sqrt{m / \varepsilon}$ is the characteristic LJ time.

Our computational domain includes a liquid film in contact with a solid sphere, which is in turn rigidly fixed on a solid substrate (see the dashed box in Fig.11). The solid substrate consists of 2500 atoms arranged on square lattice sites within the $x y$ plane with dimensions $L \times L=50.0 \sigma \times 50.0 \sigma$, and, therefore, the areal density is $1.0 \sigma^{-2}$. The interaction energy between fluid monomers and atoms of the lower stationary substrate is fixed to $1.0 \varepsilon$. Secondly, the solid particle consists of 4000 atoms uniformly distributed on a surface of a sphere with the radius $R=17.8 \sigma$, and, correspondingly, with the areal density of $1.0 \sigma^{-2}$. Thus, the important input parameter that controls wetting properties of the solid sphere is the LJ interaction energy between its atoms and fluid monomers, i.e., $\varepsilon_{\mathrm{wf}}$.

Next, the fluid phase is composed of 85000 monomers that altogether form 8500 polymer chains. Periodic boundary conditions are imposed in the $\hat{x}$ and $\hat{y}$ directions parallel to the stationary lower substrate. In MD simulations, the fluid phase is confined from above by the upper wall (not shown in Fig.11), which also consists of 2500 atoms that are arranged 
on a square plane with the linear size $L=50.0 \sigma$. The LJ interaction energy between fluid monomers and atoms of the upper wall is also set to $1.0 \varepsilon$. In contrast to the stationary substrate, the upper wall can move freely under forces from the fluid atoms and under the external pressure $P$, which is applied along the $\hat{z}$ direction toward the solid substrate. Thus, within this geometry, the position of the liquid film relative to the stationary substrate and shape of the liquid-vapor interface are determined by two parameters, i.e., $\varepsilon_{\mathrm{wf}}$ and $P$. We finally comment that the effects of gravity and entrapped gas were not considered in the present study.

A complimentary analysis of the liquid film trapped by the surface of a spherical particle was performed using the numerical software Surface Evolver [33]. In the continuum simulations, the liquid interface gradually evolves toward a state with a minimum energy by the gradient descent method. To facilitate the comparison, the geometry of the problem shown in Fig. 1 includes the same ratio of the sphere radius and the linear size of the simulation cell, $R / L$, as the one used in the MD setup. As discussed in the next section, the results of MD simulations for the contact angle as a function of the surface energy were used as input parameters in the continuum analysis.

\section{RESULTS}

At the atomic level, the local contact angle and shape of the vapor-liquid interface near the contact line depend on densities of liquid and solid phases and the interaction energy between fluid monomers and wall atoms [34 37]. In order to determine the local contact angle as a function of the surface energy, we first performed a set of separate MD simulations and considered a polymeric droplet in contact with a solid substrate. More specifically, the droplet was composed of 8500 bead-spring polymer chains, each of length $N_{p}=10$, which were placed in contact with a solid substrate that contains 25600 atoms rigidly fixed on a square lattice with lateral dimensions $160.0 \sigma \times 160.0 \sigma$, and, thus, with the areal density of $1.0 \sigma^{-2}$. Note that the areal density of atoms on the solid substrate is chosen to be the same as that for the solid spherical particle described in the previous section. In addition, the thermostatting procedure, interaction potentials, and the relative size of solid and fluid atoms were kept the same as in Sec.II].

Typical examples of liquid droplets partially wetting solid substrates are shown in Fig.2 
for the selected values of the wall-fluid interaction energy. For each value of $\varepsilon_{\mathrm{wf}}$, the system was equilibrated at the temperature of $1.0 \varepsilon / k_{B}$ for at least $3 \times 10^{6} \mathrm{MD}$ steps. It can be clearly observed that with increasing surface energy, the shape of the liquid-vapor interface becomes less curved and the liquid-solid contact area increases. We comment that with further increasing wall-fluid interaction energy, $\varepsilon_{\mathrm{wf}} \geqslant 1.0 \varepsilon$, the solid substrate becomes uniformly covered with a flat liquid film with thickness of about $3-4 \sigma$ (not shown). The contact angle was extracted by fitting a spherical cap to a liquid droplet and then averaged over several independent configurations for each value of $\varepsilon_{\mathrm{wf}}$. The summary of the data is shown in Fig. 3 for different the wall-fluid interaction energies. As expected, the local contact angle, $\theta$, decreases monotonically from a large value of about $164.1^{\circ}$, in the case of droplet on a nearly nonwetting substrate to zero for a thin liquid film. These values of the local contact angle were used as input parameters to model the shape of liquid interfaces around a spherical particle using the energy minimization method implemented in the software Surface Evolver (described below).

We now turn our discussion to the analysis of partially wetting states on textured surfaces covered by spherical particles. To make the problem computationally feasible, only one spherical particle with periodic boundary conditions in lateral directions was considered, as illustrated in Fig.1. The spherical particle is attached rigidly to the solid substrate, whose atoms interact with the fluid monomers with the LJ energy of $1.0 \varepsilon$. Two series of snapshots extracted from MD simulations are displayed in Fig. 4 for $\varepsilon_{\mathrm{wf}}=0.3 \varepsilon$ and in Fig. 5 for $\varepsilon_{\mathrm{wf}}=0.6 \varepsilon$. In each case, the external pressure was applied on the upper wall (not shown) along the negative $\hat{z}$ direction. The liquid film and its interface were allowed to relax during the time interval of $5 \times 10^{3} \tau$ each time the pressure was incremented by $0.05 \varepsilon \sigma^{-3}$. Notice the finite thickness of the liquid-vapor interface where some chain segments temporarily move away due to thermal fluctuations.

At zero applied pressure, the liquid-vapor interface is flat and its location relative to the lower substrate is determined by the local contact angle, $\theta\left(\varepsilon_{\mathrm{wf}}\right)$, at the surface of the solid sphere [see Fig.4(a) and Fig.5(a)]. We checked that this local contact angle is the same as the contact angle measured for a polymer droplet on a flat substrate. According to the results in Fig.3, the local contact angle is larger at lower surface energy, and, therefore, the liquid interface is located further away from the solid substrate in the case $\varepsilon_{\mathrm{wf}}=0.3 \varepsilon$. 
It is clearly seen in Figs. 4 and 5 that upon increasing external pressure, the liquid film is displaced closer to the solid substrate and the liquid-vapor interface becomes more curved, especially near the corners of the simulation cell that are located further away from the spherical particle and its periodic images.

We find that for each value of the wall-fluid interaction energy, when the pressure is increased up to a critical value, the sagged liquid/vapor interface touches the solid substrate, which triggers a transition to a fully wetting Wenzel state. Once the liquid film completely wets the solid substrate and the spherical particle, it remains in the wetting state even if the external pressure is reduced. In other words, the wetting transition is irreversible upon decreasing pressure down to zero. Furthermore, the variation of the critical pressure as a function of the contact angle is presented in Fig.6. To remind, the values of the local contact angle at the surface of the spherical particle were estimated from the shape of the interface of a liquid droplet residing on a flat substrate, which has the same density as the particle (see Fig.37. As evident in Fig.6, the critical pressure increases for more nonwetting particles. In the vicinity of the wetting transition, the external pressure was incremented in smaller steps, by $0.01 \varepsilon \sigma^{-3}$, in order to resolve the critical pressure more accurately. Hence, for each value of $\theta$, the maximum applied pressure at which the liquid interface remains suspended at the particle surface is also included in Fig.6.

An alternative approach to estimate the critical pressure and the shape of threedimensional liquid interfaces at structured surfaces involves an energy minimization implemented in the software Surface Evolver [33]. In this method, the surfaces of the lower substrate, spherical particle, and liquid interface are represented as a simplicial complex that consists of vertices, edges and facets. Similar to the MD setup, periodic boundary conditions were applied in the directions parallel to the solid substrate in order to mimic a periodic array of spherical particles. Furthermore, the ratio of the particle radius to the linear size of the lower substrate was chosen to be the same as in MD simulations, $R / L=0.366$, where a finite size of a liquid monomer was taken into account. The liquid film was initially placed above the spherical particle parallel to the solid substrate, and the liquid interface was allowed to evolve iteratively to the state with the lowest energy using the gradient descent method. As in MD simulations, the liquid volume is sufficiently large so that the spherical particle can be completely immersed into the liquid phase in the Wenzel state. At each step 
during the iteration procedure, the liquid interface is relaxed to form the prescribed local contact angle with the surface of the spherical particle. Note that the same values of the local contact angle as reported in Fig. 3 were used in the continuum simulations.

Typical snapshots of liquid/vapor interfaces around the spherical particle are shown in Fig.7 for $\theta=94.86^{\circ}$ and in Fig. 8 for $\theta=138.94^{\circ}$ for the indicated values of the applied pressure. It can be observed that the liquid interface is flat in the absence of the applied pressure, and it becomes more curved and displaced towards the solid substrate at higher pressures. Upon further increasing external pressure, the liquid first touches the solid substrate at the corners of the simulation cell and then spreads at the substrate until the interface forms a prescribe contact angle with the solid wall (not shown). The critical pressure of the wetting transition was determined with the accuracy of $0.5 \mathrm{~Pa}$.

The results for the critical pressure for different contact angles obtained from MD simulations and energy minimization are summarized in Fig.9. In order to compare the simulation results, we used the dimensionless variable $P_{c r} L / \gamma$, where $L$ is the linear size of the solid substrate and $\gamma$ is the surface tension coefficient. In the Surface Evolver, the input parameters were explicitly set to $L=2.73 \mathrm{~mm}$ and $\gamma=1.0 \mathrm{~N} / \mathrm{m}$. By contrast, in MD simulations the local contact angles and the surface tension are determined by the LJ interaction parameters and need to be measured separately. In the previous study, the surface tension was estimated to be $\gamma=0.85 \varepsilon / \sigma^{2}$ for a thin liquid film that consists of bead-spring 10-mers at $T=1.0 \varepsilon / k_{B}$ and zero ambient pressure [38].

It can be seen in Fig. 9 that the critical pressure obtained from MD simulations agree well with the continuum predictions. These results imply that a continuum stability analysis of partially wetting states on nanotextured surfaces might hold at length scales of about a few nanometers. Thus, in our setup the closest distance between the surface of the spherical particle and its images is about 30 molecular diameters. It should be also mentioned that one contributing factor to the slight discrepancy between two approaches involves a finite cutoff radius of the LJ interaction potential. In other words, if the sagged liquid/vapor interface is located within the cutoff radius from the solid substrate, then a spontaneous wetting of the substrate might occur due to thermal fluctuations. We finally comment that only one value of the areal fraction was considered in the present study, i.e., $\phi_{S}=\pi R^{2} / L^{2}=0.42$. It is expected that with decreasing areal fraction, the critical pressure is reduced due to smaller 
curvature of the liquid/vapor interface at the contact with the solid substrate [15].

\section{CONCLUSIONS}

In summary, molecular dynamics simulations and numerical minimization of the interfacial energy were carried out to study wetting properties of the composite interface that consists of a periodic array of spherical nanoparticles rigidly attached to a solid substrate. The wettability of spherical particles was controlled via the strength of the fluid-solid interaction energy, which in turn determines the local contact angle. It was shown that at zero applied pressure, the liquid film is suspended at the surface of solid particles and the distance to the solid substrate is determined by the particle radius and the local contact angle. Upon increasing external pressure, the liquid film is displaced closer to the solid substrate but remains stable up to a critical value due to re-entrant curvature of the particle surface. It was found that the shape of liquid interfaces and the critical pressure of permeation to the solid substrate obtained from atomistic simulations agree well with the results of the numerical minimization of the interfacial energy. These results are important for modeling partially wetting states on hierarchically textured surfaces that contain surface roughness on multiple length scales.

\section{Acknowledgments}

Financial support from the National Science Foundation (CNS-1531923) is gratefully acknowledged. The molecular dynamics simulations were carried out using the LAMMPS numerical code [30]. The analysis of liquid interfaces was performed using publicly available computer program Surface Evolver developed by Prof. K. A. Brakke at Susquehanna University. Computational work in support of this research was performed at Michigan State University's High Performance Computing Facility and the Ohio Supercomputer Center.

[1] M. N. MacGregor-Ramiasa and K. Vasilev, Questions and answers on the wettability of nanoengineered surfaces, Adv. Mater. Interfaces 4, 1700381 (2017). 
[2] X.-M. Li, D. Reinhoudt, and M. Crego-Calama, What do we need for a superhydrophobic surface? A review on the recent progress in the preparation of superhydrophobic surfaces, Chem. Soc. Rev. 36, 1350 (2007).

[3] T. L. Sun, L. Feng, X. F. Gao, and L. Jiang, Bioinspired surfaces with special wettability, Acc. Chem. Res. 38, 644 (2005).

[4] J. Genzer and K. Efimenko, Recent developments in superhydrophobic surfaces and their relevance to marine fouling: a review, Biofouling 22, 339 (2006).

[5] C. Lee and C.-J. Kim, Underwater restoration and retention of gases on superhydrophobic surfaces for drag reduction, Phys. Rev. Lett. 106, 014502 (2011).

[6] O. I. Vinogradova and A. L. Dubov, Superhydrophobic textures for microfluidics, Mendeleev Commun. 22, 229 (2012).

[7] T. Lee, E. Charrault, and C. Neto, Interfacial slip on rough, patterned and soft surfaces: A review of experiments and simulations, Adv. Colloid Interface Sci. 21, 210 (2014).

[8] C. Ybert, C. Barentin, C. Cottin-Bizonne, P. Joseph, and L. Bocquet, Achieving large slip with superhydrophobic surfaces: Scaling laws for generic geometries, Phys. Fluids 19, 123601 (2007).

[9] M. Z. Bazant and O. I. Vinogradova, Tensorial hydrodynamic slip, J. Fluid Mech. 613, 125 (2008).

[10] N. V. Priezjev, A. A. Darhuber, and S. M. Troian, Slip behavior in liquid films on surfaces of patterned wettability: Comparison between continuum and molecular dynamics simulations, Phys. Rev. E 71, 041608 (2005).

[11] N. V. Priezjev, Molecular diffusion and slip boundary conditions at smooth surfaces with periodic and random nanoscale textures, J. Chem. Phys. 135, 204704 (2011).

[12] R. N. Wenzel, Resistance of solid surfaces to wetting by water, Ind. Eng. Chem. 28, 988 (1936).

[13] A. B. D. Cassie and S. Baxter, Wettability of porous surfaces, Trans. Faraday Soc. 40, 546 (1944).

[14] A. Tuteja, W. Choi, M. Ma, J. M. Mabry, S. A. Mazzella, G. C. Rutledge, G. H. McKinley, and R. E. Cohen, Designing superoleophobic surfaces, Science 318, 1618 (2007).

[15] A. Tuteja, W. Choi, J. M. Mabry, G. H. McKinley, and R. E. Cohen, Robust omniphobic surfaces, Proc. Natl. Acad. Sci. USA 105, 18200 (2008). 
[16] M. Nosonovsky and B. Bhushan, Why re-entrant surface topography is needed for robust oleophobicity, Phil. Trans. R. Soc. A 374, 20160185 (2016).

[17] B. Liu and F. F. Lange, Pressure induced transition between superhydrophobic states: Configuration diagrams and effect of surface feature size, J. Colloid Interface Sci. 298, 899 (2006).

[18] H.-Y. Guo, B. Li, and X.-Q. Feng, Stability of Cassie-Baxter wetting states on microstructured surfaces, Phys. Rev. E 94, 042801 (2016).

[19] T. Koishi, K. Yasuoka, S. Fujikawa, T. Ebisuzaki, and X. C. Zeng, Coexistence and transition between Cassie and Wenzel state on pillared hydrophobic surface, Proc. Natl. Acad. Sci. USA 106, 8435 (2009).

[20] X. Yong and L. T. Zhang, Nanoscale wetting on groove-patterned surfaces, Langmuir 25, 5045 (2009).

[21] T.-H. Yen, Investigation of the effects of perpendicular electric field and surface morphology on nanoscale droplet using molecular dynamics simulation, Molecular Simulation 38, 509 (2012).

[22] J. K. Saha, M. A. Matin, J. Jang, and J. Jang, Molecular dynamics simulation study on the wetting behavior of a graphite surface textured with nanopillars, Bull. Korean Chem. Soc. 34, 1047 (2013).

[23] Q. Yuan and Y.-P. Zhao, Wetting on flexible hydrophilic pillar-arrays, Sci. Rep. 3, 1944, (2013).

[24] X. M. Xu, G. Vereecke, C. Chen, G. Pourtois, S. Armini, N. Verellen, W.-K. Tsai, D.-W. Kim, E. Lee, C.-Y. Lin, P. V. Dorpe, H. Struyf, F. Holsteyns, V. Moshchalkov, J. Indekeu, and S. De Gendt, Capturing wetting states in nanopatterned silicon, ACS Nano 8, 885 (2014).

[25] Q. Yuan and Y.-P. Zhao, Statics and dynamics of electrowetting on pillar-arrayed surfaces at the nanoscale, Nanoscale 7, 2561 (2015).

[26] A. M. Miqdad, S. Datta, A. K. Das, and P. K. Das, Effect of electrostatic incitation on the wetting mode of a nano-drop over a pillar-arrayed surface, RSC Adv. 6, 110127 (2016).

[27] W. Xu, Z. Lan, B. L. Peng, R. F. Wen, and X. H. Ma, Effect of nano structures on the nucleus wetting modes during water vapour condensation: from individual groove to nanoarray surface, RSC Adv. 6, 7923 (2016).

[28] C.-C. Chang, Y.-J. Sheng, and H.-K. Tsao, Wetting hysteresis of nanodrops on nanorough surfaces, Phys. Rev. E 94, 042807 (2016).

[29] J. Yan, K. Yang, X. Zhang, and J. Zhao, Analysis of impact phenomenon on superhydrophobic 
surfaces based on molecular dynamics simulation, Comput. Mater. Sci. 134, 8 (2017).

[30] S. J. Plimpton, Fast parallel algorithms for short-range molecular dynamics, J. Comp. Phys. 117, 1 (1995).

[31] M. P. Allen and D. J. Tildesley, Computer Simulation of Liquids (Clarendon, Oxford, 1987).

[32] K. Kremer and G. S. Grest, Dynamics of entangled linear polymer melts: A molecular dynamics simulation, J. Chem. Phys. 92, 5057 (1990).

[33] K. A. Brakke, The Surface Evolver, Experimental Mathematics 1, 141 (1992).

[34] P. van Remoortere, J. E. Mertz, L. E. Scriven, and H. T. Davis, Wetting behavior of a Lennard-Jones system, J. Chem. Phys. 110, 2621 (1999).

[35] A. Milchev, A. Milchev, and K. Binder, Nanodroplets on a solid plane: wetting and spreading in a Monte Carlo simulation, Comput. Phys. Comm. 146, 38 (2002).

[36] E. Bertrand, T. D. Blake, and J. De Coninck, Influence of solid-liquid interactions on dynamic wetting: a molecular dynamics study, J. Phys.: Condens. Matter 21, 464124 (2009).

[37] J. H. Weijs, A. Marchand, B. Andreotti, D. Lohse, and J. H. Snoeijer, Origin of line tension for a Lennard-Jones nanodroplet, Phys. Fluids 23, 022001 (2011).

[38] D. R. Heine, G. S. Grest, and E. B. Webb, Spreading dynamics of polymer nanodroplets, Phys. Rev. E 68, 061603 (2003). 
Figures 


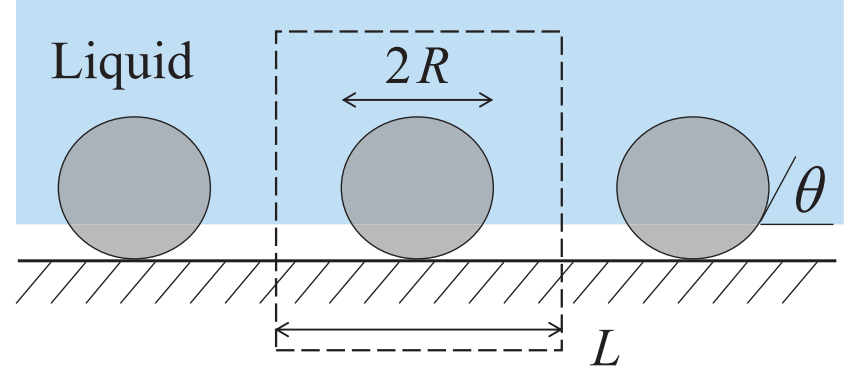

FIG. 1: (Color online) A schematic illustration of the structured interface that consists of an array of spherical particles attached to the solid substrate and a suspended liquid film. The local contact angle of the liquid interface at the surface of the solid particle is denoted by $\theta$. The dashed box indicates the spatial domain used in molecular dynamics and continuum simulations. 
(a)

(b)
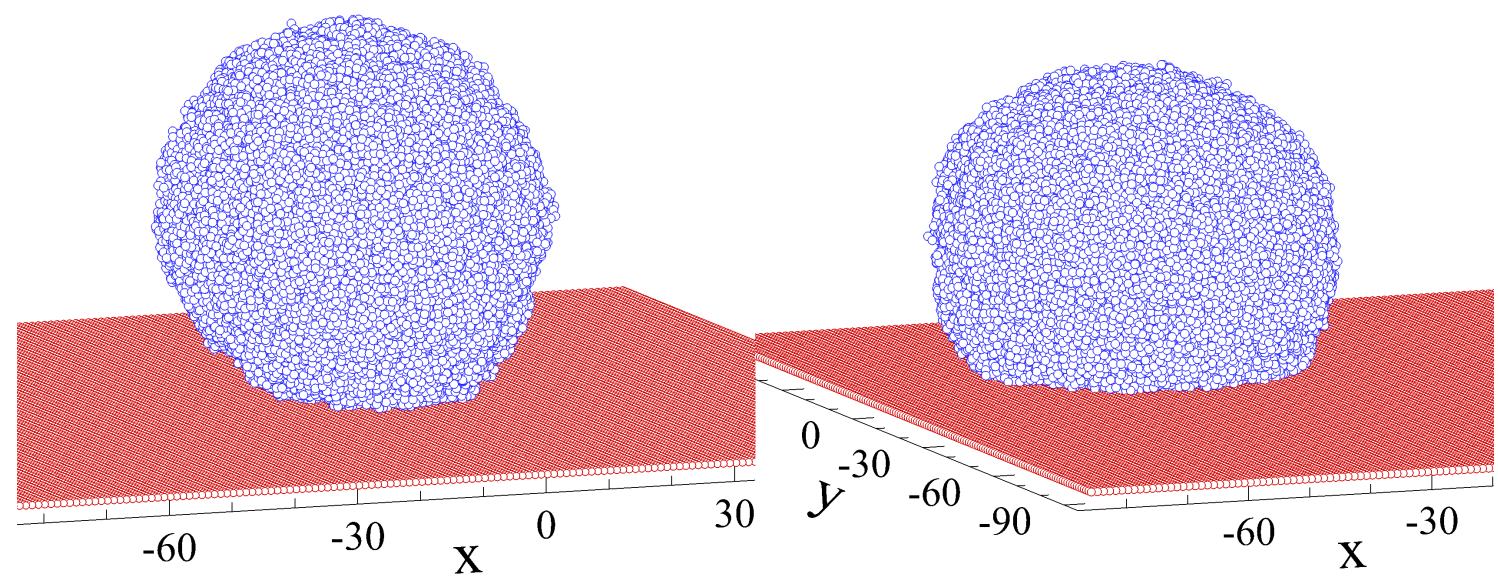

(c)

(d)

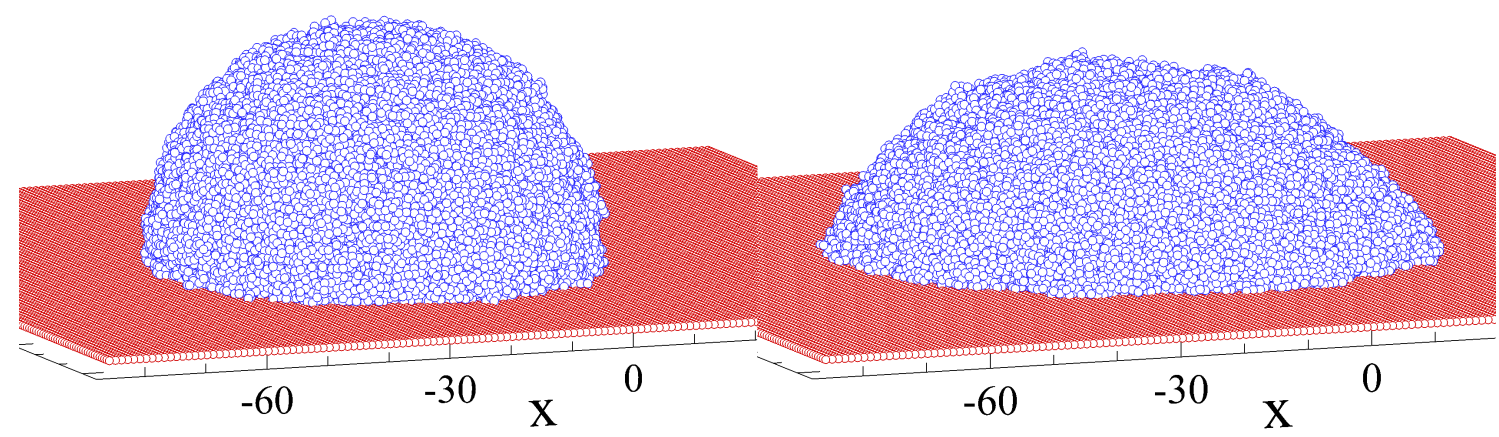

FIG. 2: (Color online) Snapshots of liquid droplets that consist of 85000 atoms (blue circles) in contact with the solid substrate (red circles) with the wall-fluid interaction energy (a) $\varepsilon_{\mathrm{wf}}=0.2 \varepsilon$, (b) $\varepsilon_{\mathrm{wf}}=0.4 \varepsilon$, (c) $\varepsilon_{\mathrm{wf}}=0.6 \varepsilon$, and (d) $\varepsilon_{\mathrm{wf}}=0.8 \varepsilon$. The dimensions of the solid substrate are $160.0 \sigma \times 160.0 \sigma$. 


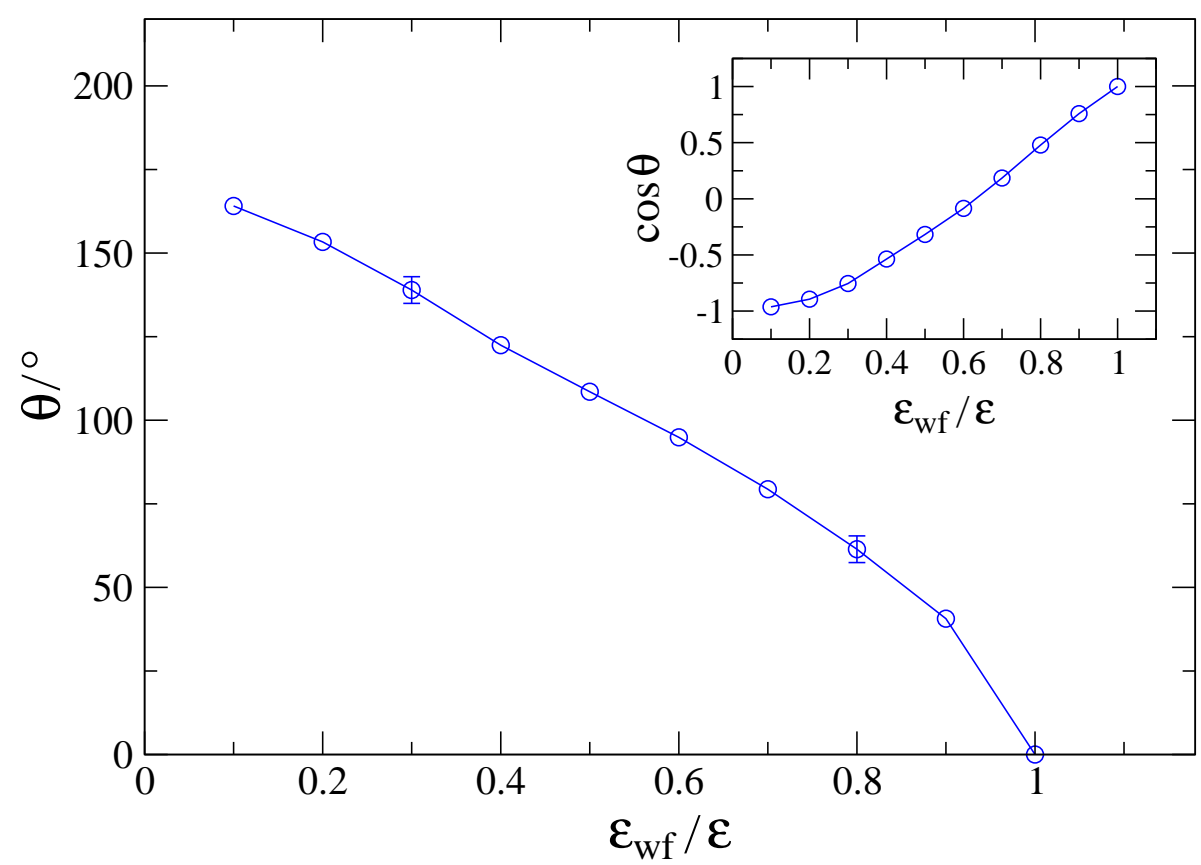

FIG. 3: (Color online) The dependence of the contact angle as a function of the surface energy $\varepsilon_{\mathrm{wf}} / \varepsilon$ for liquid droplets residing on a crystalline substrate. The data are obtained from the best fit of the liquid-vapor interface to a spherical cap. The inset shows the same data replotted as $\cos \theta\left(\varepsilon_{\mathrm{wf}}\right)$. 

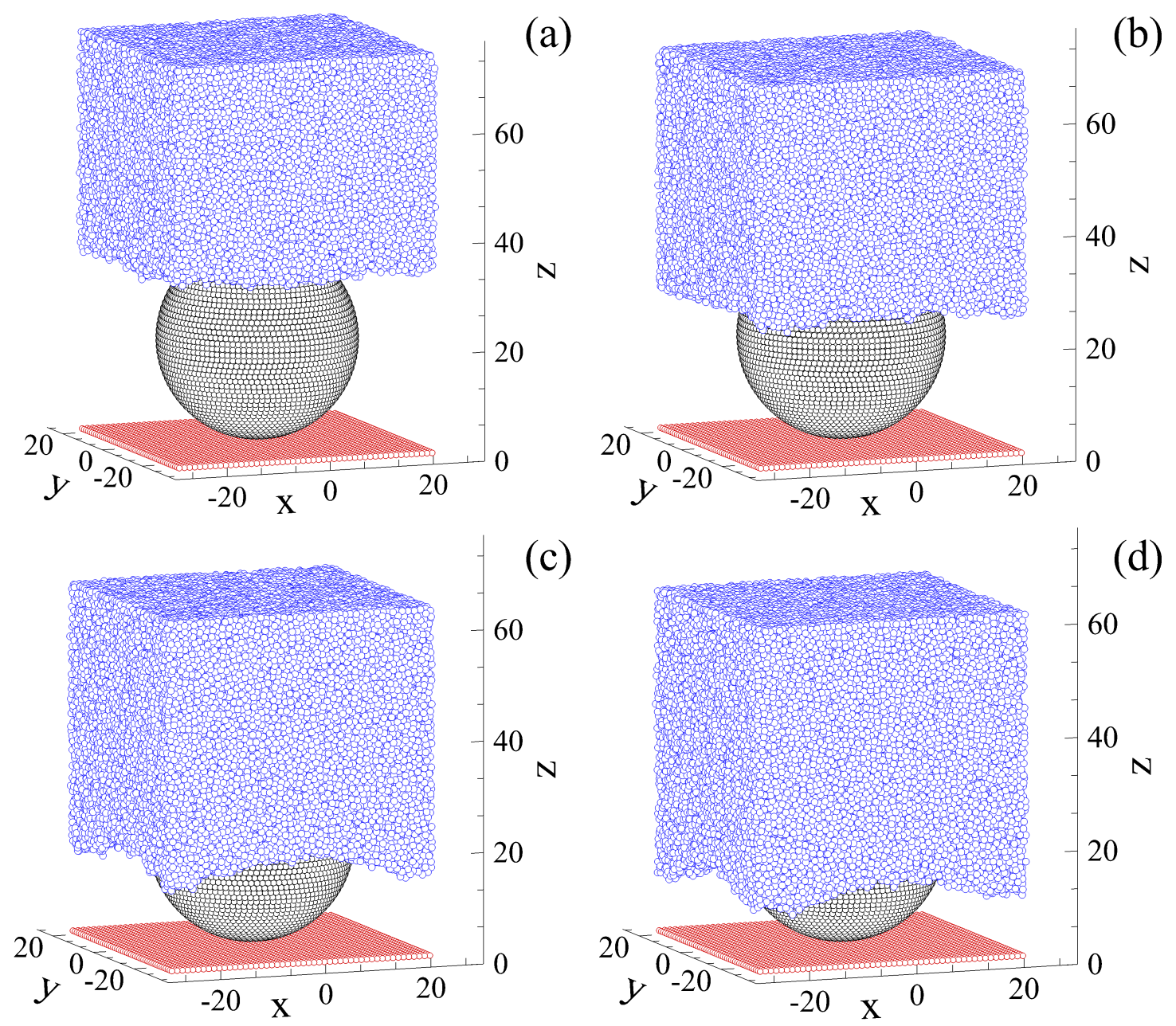

FIG. 4: (Color online) Snapshots of the liquid film suspended on the spherical particle for the wall-fluid interaction energy $\varepsilon_{\mathrm{wf}}=0.3 \varepsilon$ and the vertical pressure (a) $P=0$, (b) $P=0.02 \varepsilon \sigma^{-3}$, (c) $P=0.05 \varepsilon \sigma^{-3}$, and (d) $P=0.055 \varepsilon \sigma^{-3}$. The averaged value of the local contact angle is $\theta=138.94^{\circ}$. 
(a)
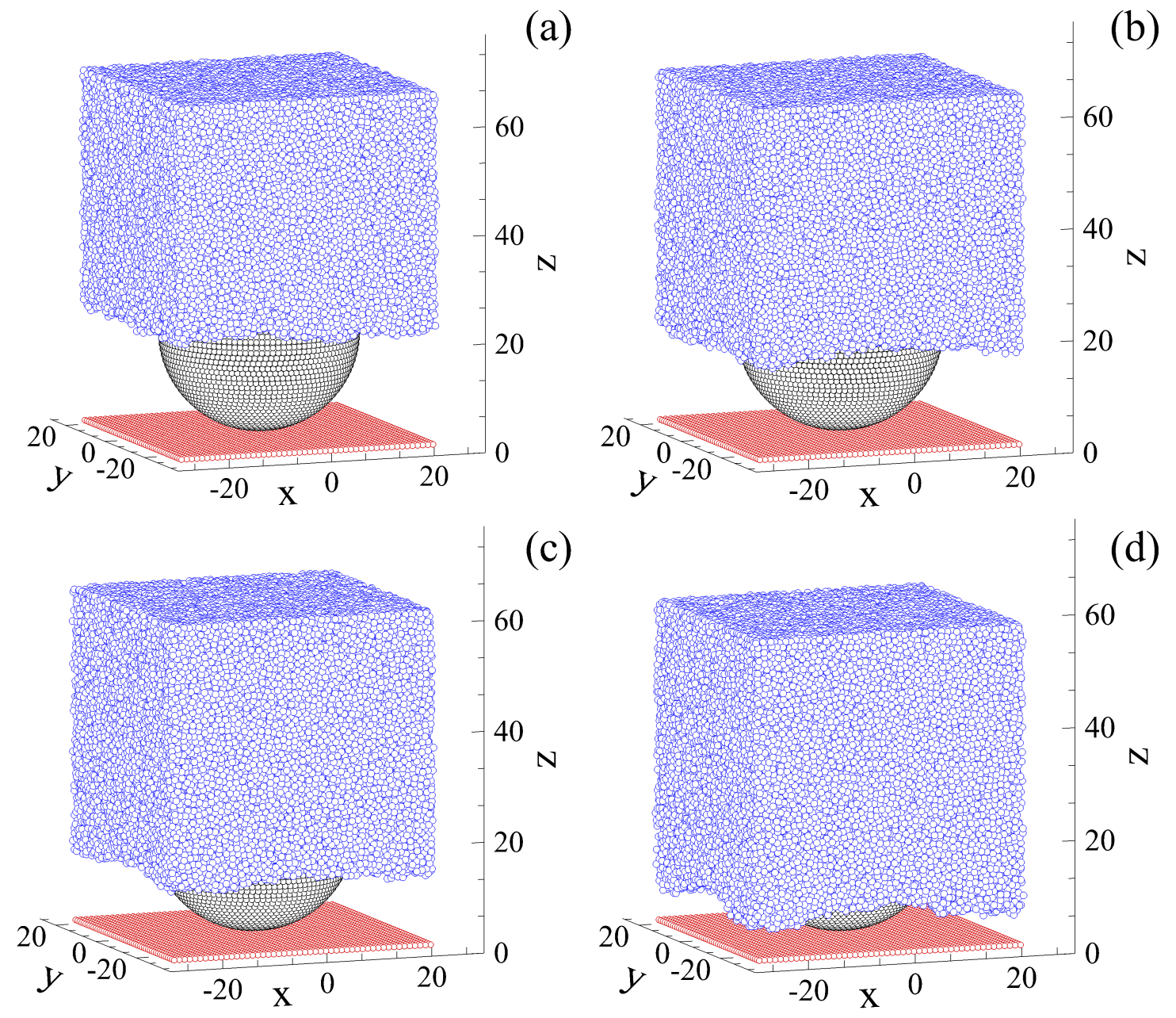

FIG. 5: (Color online) A liquid film partially wetting the spherical particle for the wall-fluid interaction energy $\varepsilon_{\mathrm{wf}}=0.6 \varepsilon$ and the vertical pressure (a) $P=0$, (b) $P=0.01 \varepsilon \sigma^{-3}$, (c) $P=$ $0.02 \varepsilon \sigma^{-3}$, and (d) $P=0.03 \varepsilon \sigma^{-3}$. The local contact angle at the particle surface is $\theta=94.86^{\circ}$. 


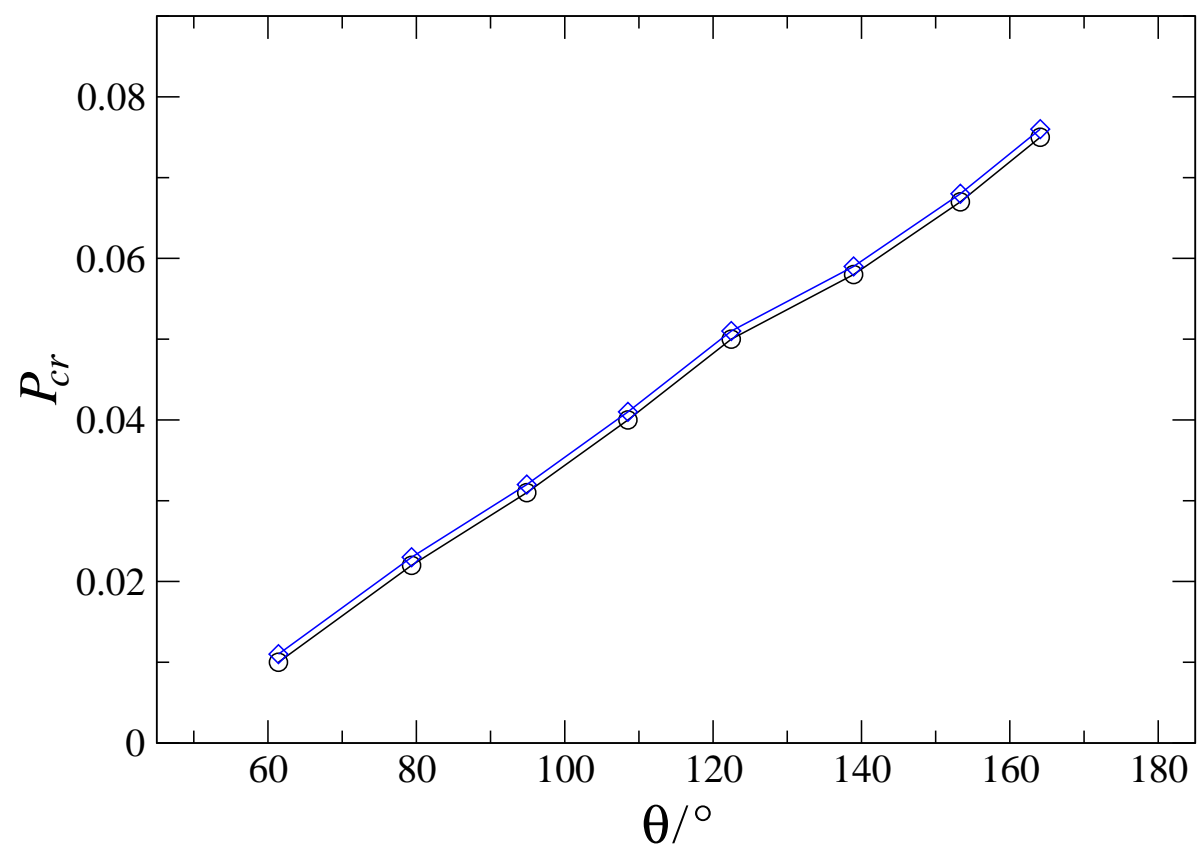

FIG. 6: (Color online) The critical pressure $P_{c r}$ (in units of $\varepsilon \sigma^{-3}$ ) as a function of the local contact angle $\theta$ (in degrees). The largest external pressure at which the liquid interface does not touch the lower substrate is denoted by $(\circ)$. The threshold pressure associated with a wetting transition to the Wenzel state is indicated by $(\diamond)$. 


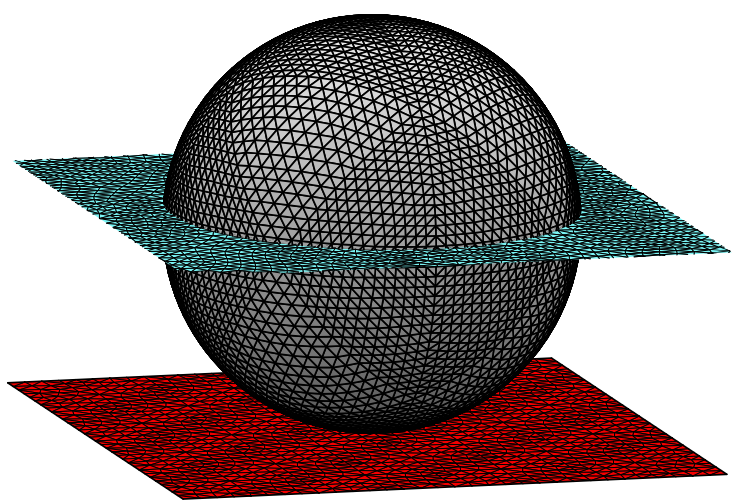

(a)

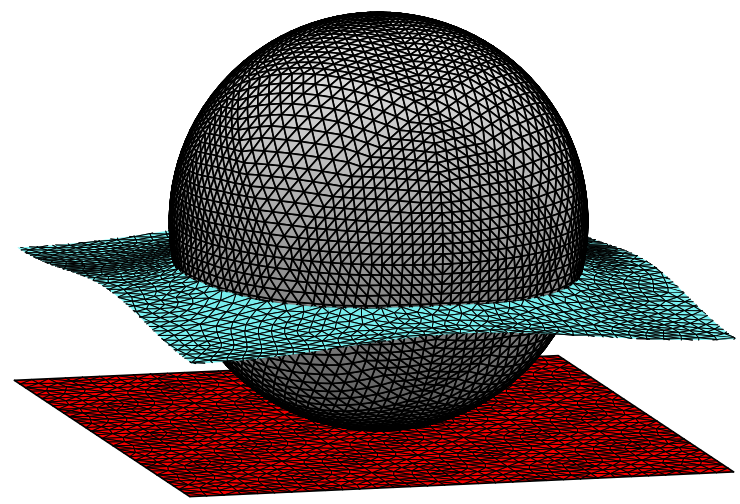

(c)

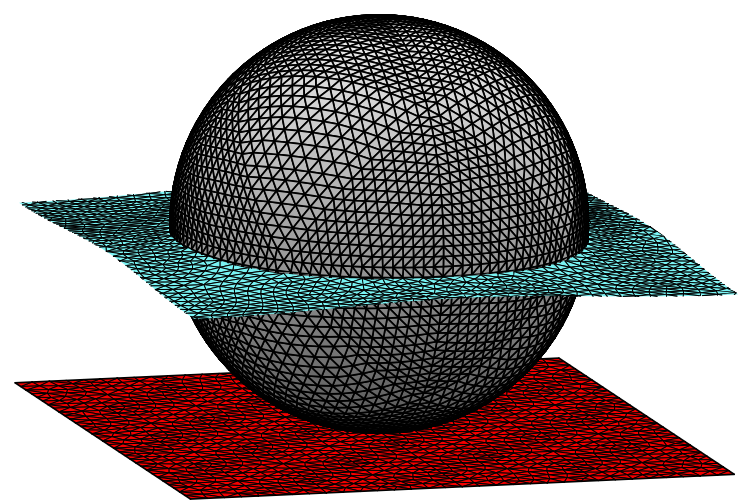

(b)

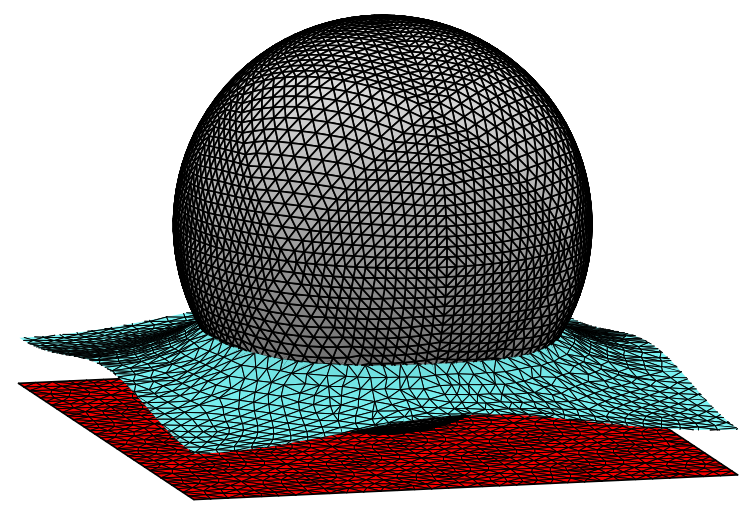

(d)

FIG. 7: (Color online) A sequence of snapshots of the liquid/vapor interface obtained numerically using the software Surface Evolver. The local contact angle at the surface of the solid sphere is $\theta=94.86^{\circ}$ and the surface tension coefficient is $\gamma=1.0 \mathrm{~N} / \mathrm{m}$. The external pressure (a) $P=0$, (b) $P=205 \mathrm{~Pa}$, (c) $P=410 \mathrm{~Pa}$, and (d) $P=610 \mathrm{~Pa}$. 


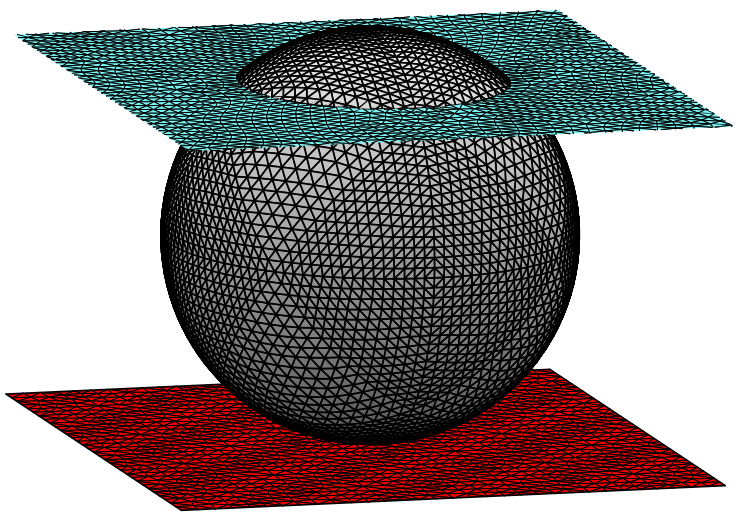

(a)

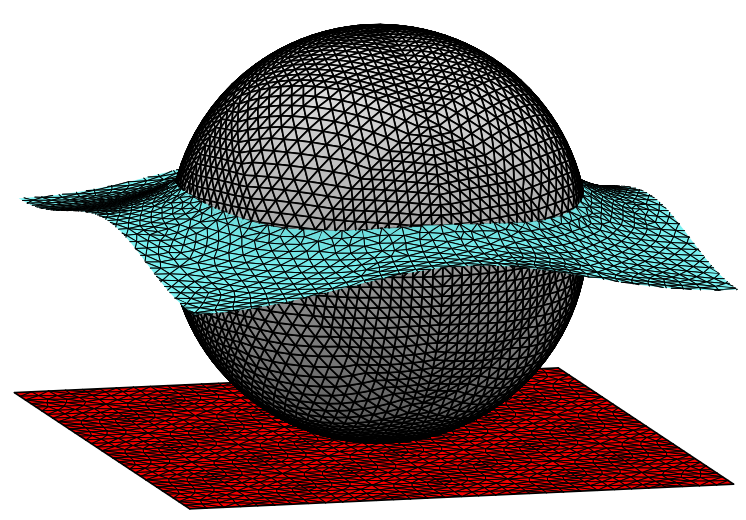

(c)

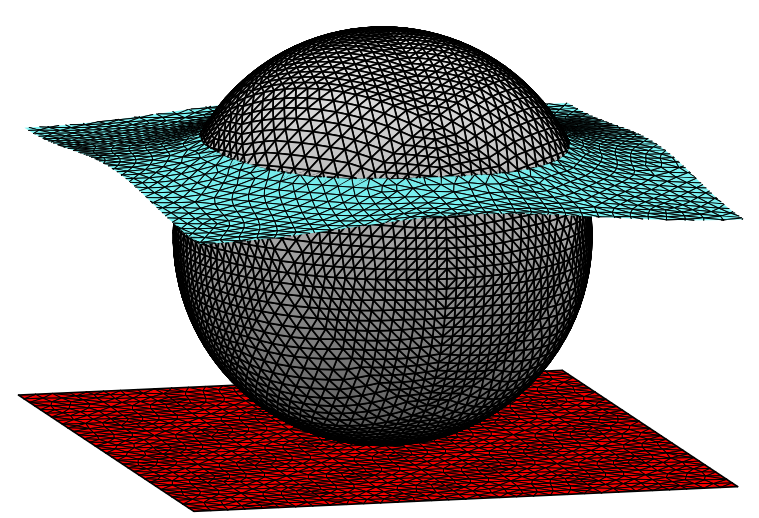

(b)

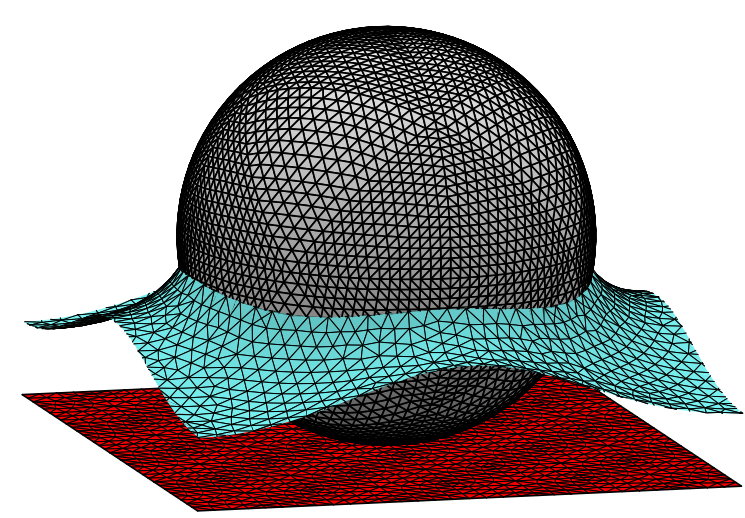

(d)

FIG. 8: (Color online) The shape of liquid/vapor interfaces for the external pressures (a) $P=0$, (b) $P=400 \mathrm{~Pa}$, (c) $P=800 \mathrm{~Pa}$, and (d) $P=1200 \mathrm{~Pa}$. The contact angle at the surface of the spherical particle is $\theta=138.94^{\circ}$ and the surface tension of the liquid/vapor interface is $\gamma=1.0 \mathrm{~N} / \mathrm{m}$. 


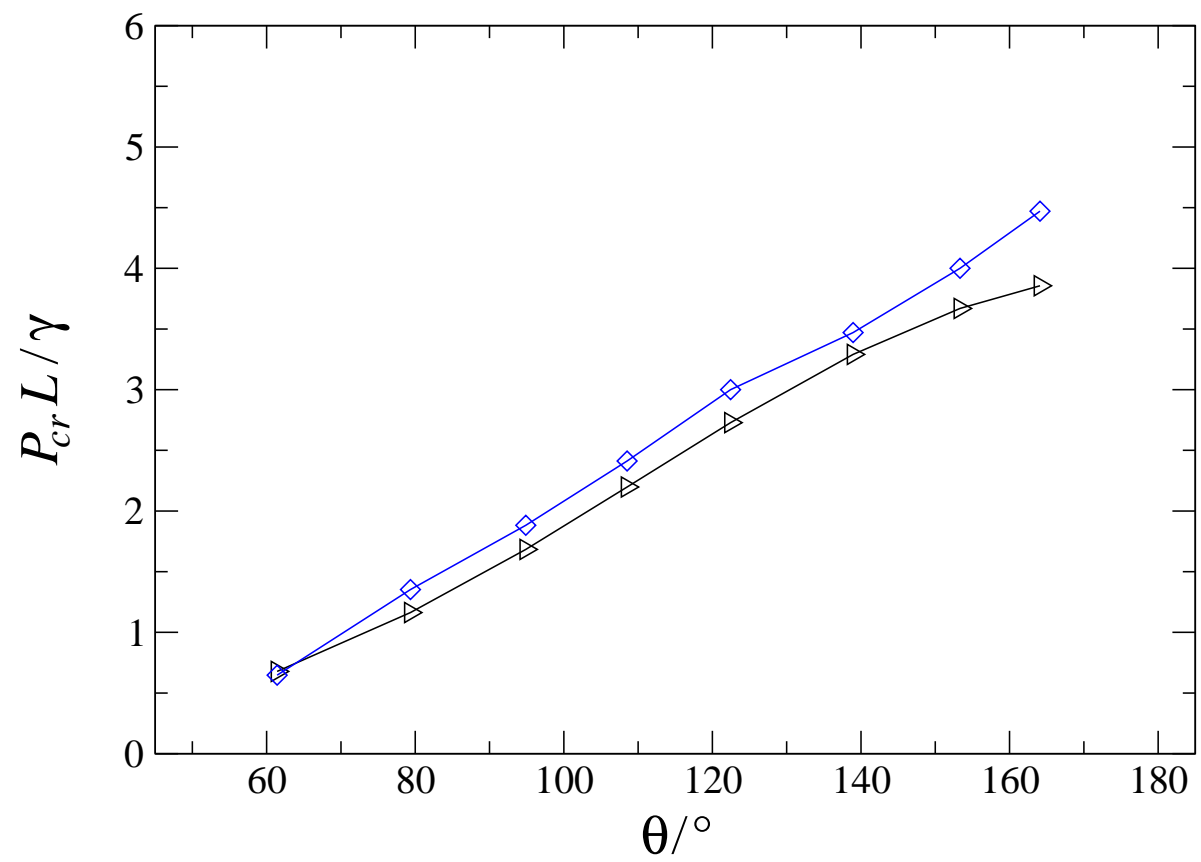

FIG. 9: (Color online) The dimensionless variable $P_{c r} L / \gamma$ as a function of the local contact angle $\theta$. The MD data are indicated by $(\diamond)$ and continuum results are denoted by $(\triangleright)$. Here, $L$ is the linear size of the lower substrate and $\gamma$ is the surface tension of the liquid/vapor interface. The error bars are about the symbol size. 